\title{
Hypergeometric summation revisited
}

\author{
S. A. Abramov ${ }^{*}$ \\ Russian Academy of Sciences \\ Dorodnicyn Computing Centre \\ Vavilova 40, 119991, Moscow GSP-1, Russia \\ sabramov@ccas.ru \\ M. Petkovšek ${ }^{\dagger}$ \\ Faculty of Mathematics and Physics \\ University of Ljubljana, \\ Jadranska 19, SI-1000 Ljubljana, Slovenia \\ marko.petkovsek@uni-1j.si
}

\begin{abstract}
We consider hypergeometric sequences, i.e., the sequences which satisfy linear first-order homogeneous recurrence equations with relatively prime polynomial coefficients. Some results related to necessary and sufficient conditions are discussed for validity of discrete NewtonLeibniz formula $\sum_{k=v}^{w} t(k)=u(w+1)-u(v)$ when $u(k)=R(k) t(k)$ and $R(k)$ is a rational solution of Gosper's equation.
\end{abstract}

\section{Introduction}

Let $K$ be a field of characteristic zero ( $K=\mathbb{C}$ in all examples). If $t(k) \in$ $K(k)$ then the telescoping equation

$$
u(k+1)-u(k)=t(k)
$$

may or may not have a rational solution $u(k)$, depending on the type of $t(k)$. Here the telescoping equation is considered as an equality in the rationalfunction field, regardless of the possible integer poles that $u(k)$ and/or $t(k)$ might have.

\footnotetext{
*Partially supported by RFBR under grant 04-01-00757.

'Partially supported by MZT RS under grant J2-8549.
} 
An algorithm for finding rational $u(k)$ was proposed in 1971 (see [1]). It follows from that algorithm that if $t(k)$ has no integer poles, then a rational $u(k)$ satisfying (1), if it exists, has no integer poles either, and the discrete Newton-Leibniz formula

$$
\sum_{k=v}^{w} t(k)=u(w+1)-u(v)
$$

is valid for any integer bounds $v \leq w$. Working with polynomial and rational functions we will write $f(k) \perp g(k)$ for $f(k), g(k) \in K[k]$ to indicate that $f(k)$ and $g(k)$ are coprime; if $R(k) \in K(k)$, then $\operatorname{den}(R(k))$ is the monic polynomial from $K[k]$ such that $R(k)=\frac{f(k)}{\operatorname{den}(R(k))}$ for some $f(k) \in K[k], f(k) \perp \operatorname{den}(R(k))$.

The problem of solving equation (1) can be considered for sequences. If $t(k)$ is a sequence, we use the symbol $E$ for the shift operator w.r. to $k$, so that $E t(k)=t(k+1)$. In the rest of the paper we assume that the sequences under consideration are defined on an infinite interval $I$ of integers and either $I=\mathbb{Z}$, or

$$
I=\mathbb{Z}_{\geq l}=\{k \in \mathbb{Z} \mid k \geq l\}, l \in \mathbb{Z} .
$$

If a sequence $t(k)$ defined on $I$ is given, and a sequence $u(k)$, which is also defined on $I$ and satisfies (1) for all $k \in I$, is found (any such sequence is a primitive of $t(k)$ ), then we can use formula (2) for any $v \leq w$ with $v, w \in I$.

Gosper's algorithm [6], which we denote hereafter by $\mathcal{G} \mathcal{A}$, discovered in 1978 , focuses on the case where a given $t(k)$ and an unknown $u(k)$ are hypergeometric sequences.

Definition $1 A$ sequence $y(k)$ defined on an infinite interval I is hypergeometric if it satisfies the equation $L y(k)=0$ for all $k \in I$, with

$$
L=a_{1}(k) E+a_{0}(k) \in K[k, E], \quad a_{1}(k) \perp a_{0}(k) .
$$

$\mathcal{G} \mathcal{A}$ starts by constructing the operator $L$ for a given concrete hypergeometric sequence $t(k)$, and this step is not formalized. On the next steps $\mathcal{G} \mathcal{A}$ works with $L$ only, while the sequence $t(k)$ itself is ignored (more precisely, in the case of $L=a_{1}(k) E+a_{0}(k), \mathcal{G A}$ works with the certificate of $t(k)$, i.e., with the rational function $-\frac{a_{0}(k)}{a_{1}(k)}$, but this is not essential). The algorithm tries to construct a rational function $R(k)$, which is a solution in $K(k)$ of Gosper's equation

$$
a_{0}(k) R(k+1)+a_{1}(k) R(k)=-a_{1}(k)
$$


(such $R(k)$, when it exists, can also be found by general algorithms from $[2,3])$. If such $R(k)$ exists then

$$
R(k+1) t(k+1)-R(k) t(k)=t(k)
$$

is valid for almost all integers $k$. The fact is that even when $t(k)$ is defined everywhere on $I$, it can happen that $R(k)$ has some poles belonging to $I$, and $u(k)=R(k) t(k)$ cannot be defined in such a way as to make (1) valid for all integers from $I$. One can encounter the situation where formula (2) is not valid even when all of

$$
t(v), t(v+1), \ldots, t(w), \quad u(v), u(w+1)
$$

are well-defined. The reason is that (1) may fail to hold at certain points $k$ of the summation interval. However, sometimes it is possible to define the values of $u(k)=R(k) t(k)$ appropriately for all integers $k$, even though $R(k)$ has some integer poles. In such well-behaved cases (2) can be used to compute $\sum_{k=v}^{w} t(k)$ for any $v \leq w, v, w \in I$.

Example 1 Gosper's equation, corresponding to $L=k E-(k+1)^{2}$, has a solution $R=\frac{1}{k}$. The sequences

$$
t_{1}(k)= \begin{cases}0, & \text { if } k<0 \\ k \cdot k !, & \text { if } k \geq 0\end{cases}
$$

and

$$
t_{2}(k)= \begin{cases}\frac{(-1)^{k} k}{(-k-1) !}, & \text { if } k<0 \\ 0, & \text { if } k \geq 0\end{cases}
$$

both satisfy $L y=0$ on $I=\mathbb{Z}$.

Generally speaking, (2) is not applicable to $t_{1}(k)$, but is applicable to $t_{2}(k)$. We can illustrate this as follows. Applying $(2)$ to $t_{1}(k)$ with $v=$ $-1, w=1$, we have

$$
t_{1}(-1)+t_{1}(0)+t_{1}(1)=\left.\frac{1}{k} t_{1}(k)\right|_{k=2}-\left.\frac{1}{k} t_{1}(k)\right|_{k=-1}=\frac{1}{2} \cdot 4-0=2
$$

which is wrong, because $t_{1}(-1)+t_{1}(0)+t_{1}(1)=0+0+1=1$. Applying (2) to $t_{2}$ with the same $v, w$, we have

$$
t_{2}(-1)+t_{2}(0)+t_{2}(1)=\left.\frac{1}{k} t_{2}(k)\right|_{k=2}-\left.\frac{1}{k} t_{2}(k)\right|_{k=-1}=0-(-1)=1
$$

which is correct, because $t_{2}(-1)+t_{2}(0)+t_{2}(1)=1+0+0=1$. 
In this paper we discuss some results related to necessary and sufficient conditions for validity of formula (2) when $u(k)=R(k) t(k)$, and $R(k)$ is a rational solution of corresponding Gosper's equation. If such $R(k)$ exists, then we describe the linear space of all hypergeometric sequences $t(k)$ that are defined on $I$ and such that formula (2) is valid for $u=R t$ and any integer bounds $v \leq w$ such that $v, w \in I$. The dimension of this space is always positive (it can be even bigger than 1 ). We will denote

- by $\mathcal{H}_{I}$ the set of all hypergeometric sequences defined on $I$;

- by $\mathcal{L}$ the set of all operators of type (3);

- by $V_{I}(L)$, where $L \in \mathcal{L}$, the $K$-linear space of all sequences $t(k)$ defined on $I$ for which $L t(k)=0$ for all $k \in I$;

- by $W_{I}(R(k), L)$, where $L \in \mathcal{L}$ and $R(k) \in K(k)$ is a solution of the corresponding Gosper's equation, the $K$-linear space of all $t(k) \in V_{I}(L)$ such that (2) with $u(k)=R(k) t(k)$ is valid for all $v \leq w$ with $v, w \in I$.

The paper is a summary of the results that have been published in $[4,5]$. In addition we consider the case where Gosper's equation has non-unique rational solution (Section 3.2). In Section 2 we consider individual hypergeometric sequences while in Section 3 we concentrate on spaces of the type $W_{I}(R(k), L)$.

\section{Validity conditions of the discrete Newton- Leibniz formula}

\subsection{A criterion}

Theorem 1 ([4,5]) Let $L \in \mathcal{L}, t(k) \in V_{I}(L)$, and let Gosper's equation corresponding to $L$ have a solution $R(k) \in K(k)$, with $\operatorname{den}(R)=g(k)$. Then $t(k) \in W_{I}(R(k), L)$ iff there exists a $\bar{t}(k) \in \mathcal{H}_{I}$ such that $t(k)=g(k) \bar{t}(k)$ for all $k \in I$.

Example 2 Consider again the sequences $t_{1}(k), t_{2}(k)$ on $I=\mathbb{Z}$ from Example 1 . We have $t_{2}(k)=k \bar{t}_{2}(k)$, where

$$
\bar{t}_{2}(k)= \begin{cases}\frac{(-1)^{k}}{(-k-1) !}, & \text { if } k<0, \\ 0, & \text { if } k \geq 0\end{cases}
$$


is a hypergeometric sequence defined everywhere:

$$
E \bar{t}_{2}(k)-(k+1) \bar{t}_{2}(k)=0 .
$$

On the other hand, if $t_{1}(k)=k \bar{t}_{1}(k)$ for some sequence $\bar{t}_{1}(k)$, then

$$
\bar{t}_{1}(k)= \begin{cases}0, & \text { if } k<0 \\ \zeta, & \text { if } k=0 \\ k !, & \text { if } k>0\end{cases}
$$

where $\zeta \in \mathbb{C}$. Notice that the sequence $\bar{t}_{1}(k)$ is not hypergeometric on $\mathbb{Z}$, for any $\zeta \in \mathbb{C}$.

\subsection{Summation of proper hypergeometric sequences}

Definition 2 Following conventional notation, the rising factorial power $(\alpha)_{k}$ and its reciprocal $1 /(\beta)_{k}$ are defined for $\alpha, \beta \in K$ and $k \in \mathbb{Z}$ by

$$
\begin{aligned}
(\alpha)_{k}= \begin{cases}\prod_{m=0}^{k-1}(\alpha+m), & k \geq 0 ; \\
\prod_{m=1}^{|k|} \frac{1}{\alpha-m}, & k<0, \alpha \neq 1,2, \ldots,|k| ; \\
\text { undefined, } & \text { otherwise; }\end{cases} \\
\frac{1}{(\beta)_{k}}= \begin{cases}\prod_{m=0}^{k-1} \frac{1}{\beta+m}, & k \geq 0, \beta \neq 0,-1, \ldots, 1-k ; \\
\prod_{m=1}^{|k|}(\beta-m), & k<0 ; \\
\text { undefined, } & \text { otherwise. }\end{cases}
\end{aligned}
$$

Note that if $(\alpha)_{k}$ resp. $1 /(\beta)_{k}$ is defined for some $k \in \mathbb{Z}$, then $(\alpha)_{k+1}$ resp. $1 /(\beta)_{k-1}$ is defined for that $k$ as well. Thus $(\alpha)_{k}$ and $1 /(\beta)_{k}$ are hypergeometric sequences which satisfy

$$
(\alpha)_{k+1}=(\alpha+k)(\alpha)_{k}, \quad(\beta+k) /(\beta)_{k+1}=1 /(\beta)_{k}
$$

whenever $(\alpha)_{k}$ and $1 /(\beta)_{k+1}$ are defined. 
Example 3 Let $t(k)=(k-2)(-1 / 2)_{k} /(4 k !)$. This hypergeometric sequence is defined for all $k \in \mathbb{Z}$ (note that $t(k)=0$ for $k<0$ ) and satisfies $L t(k)=0$ for all $n \in \mathbb{Z}$ where $L=a_{1}(k) E+a_{0}(k)$ with $a_{0}(k)=-(k-1)(2 k-1)$ and $a_{1}(k)=2(k-2)(k+1)$. Gosper's equation, corresponding to $L$, has a rational solution

$$
R(k)=\frac{2 k(k+1)}{k-2} .
$$

Equation (1) indeed fails at $k=1$ and $k=2$ because $u(k)=R(k) t(k)$ is undefined at $k=2$. But if we cancel the factor $k-2$ and replace $u(k)$ by the sequence

$$
\bar{u}(k)=k(k+1) \frac{(-1 / 2)_{k}}{2 k !}
$$

then equation

$$
\bar{u}(k+1)-\bar{u}(k)=t(k)
$$

holds for all $k \in \mathbb{Z}$, and

$$
\sum_{k=v}^{w} t(k)=\bar{u}(w+1)-\bar{u}(v) .
$$

The sequence $t(k)$ from Example 3 is an instance of a proper hypergeometric sequence which we are going to define now. As it turns out, there are no restrictions on the validity of the discrete Newton-Leibniz formula for proper sequences (Theorem 2).

Definition 3 A hypergeometric sequence $t(k)$ defined on an infinite interval $I$ of integers is proper if there are

- a constant $z \in K$,

- a polynomial $p(k) \in K[k]$,

- nonnegative integers $q, r$,

- constants $\alpha_{1}, \ldots, \alpha_{q}, \beta_{1}, \ldots, \beta_{r} \in K$

such that

$$
t(k)=p(k) z^{k} \frac{\prod_{i=1}^{q}\left(\alpha_{i}\right)_{k}}{\prod_{j=1}^{r}\left(\beta_{j}\right)_{k}}
$$

for all $k \in I$. 
Theorem 2 ([4]) Let $t(k)$ be a proper hypergeometric sequence defined on $I$ and given by $(9)$. Denote $a(k)=z \prod_{i=1}^{q}\left(k+\alpha_{i}\right)$ and $b(k)=\prod_{j=1}^{r}\left(k+\beta_{j}\right)$. If a polynomial $y(k) \in K[k]$ satisfies

$$
a(k) y(k+1)-b(k-1) y(k)=p(k)
$$

and if

$$
\bar{u}(k)=y(k) z^{k} \frac{\prod_{i=1}^{q}\left(\alpha_{i}\right)_{k}}{\prod_{j=1}^{r}\left(\beta_{j}\right)_{k-1}}
$$

for all $k \in I$, then equation (7) holds for all $k \in I$, and the discrete NewtonLeibniz formula (8) is valid for all $v \leq w$, when $v, w \in I$.

Notice that (10) has a solution in $K[k]$ iff Gosper's equation, corresponding to the operator from $\mathcal{L}$, annihilating $t(k)$, has a solution in $K(k)$.

Example 4 The hypergeometric sequence

$$
t(k)=\frac{\left(\begin{array}{c}
2 k-3 \\
k
\end{array}\right)}{4^{k}}
$$

which is defined for all $k \in \mathbb{Z}$ can be written as

$$
t(k)= \begin{cases}2 s(k), & k<2, \\ s(k), & k \geq 2\end{cases}
$$

where

$$
s(k)=(2-k) \frac{(-1 / 2)_{k}}{4(1)_{k}}
$$

is the proper sequence from Example 3 . For $w \geq 1$, one should first split summation range in two

$$
\sum_{k=0}^{w} t(k)=\frac{3}{4}+\sum_{k=2}^{w} s(k)
$$

then the discrete Newton-Leibniz formula can be safely used to evaluate the sum on the right. However, applying directly (2) to (11) with (6) we obtain

$$
\sum_{k=0}^{w} t(k)=(?) \quad u(w+1)-u(0)=\frac{(w+1)(w+2)\left(\begin{array}{c}
2 w-1 \\
w+1
\end{array}\right)}{2(w-1) 4^{w}}
$$

If we assume that the value of $\left(\begin{array}{c}2 k-3 \\ k\end{array}\right)$ is 1 when $k=0$ and -1 when $k=1$ (that is natural from combinatorial point of view) then the expression on the right gives the true value of the sum only at $w=0$. 


\subsection{When the interval $I$ contains no leading integer singu- larity of $L$}

Definition 4 For a linear difference operator (3) we call $M=\max (\{k \in$ $\left.\left.\mathbb{Z} ; a_{1}(k-1)=0\right\} \cup\{-\infty\}\right)$ the maximal leading integer singularity of $L$,

Proposition 1 ([4]) Let $R(k)$ be a rational solution of (4). Then $R(k)$ has no poles larger than $M-1$.

Theorem 3 ([4]) Let $L \in \mathcal{L}, M$ be the maximal integer singularity of $L$, $l \geq M, I=\mathbb{Z}_{\geq l}$ and $t(k) \in V_{I}(L)$. Let Gosper's equation, corresponding to $L$, have a solution $R(k)$ in $K(k)$. Then $t(k) \in W_{I}(R(k), L)$.

Example 5 For the sequence (11) we have $a_{0}(k)=-(2 k-1)(k-1), a_{1}(k)=$ $2(k+1)(k-2), R(k)=2 k(k+1) /(k-2)$, and $u(k)=2 k(k+1)\left(\begin{array}{c}2 k-3 \\ k\end{array}\right) /((k-$ $\left.2) 4^{k}\right)$. Thus $M=3$, and the only pole of $R(k)$ is $k=2$. As predicted by Theorem 3 , the discrete Newton-Leibniz formula is valid when, e.g., $3 \leq v \leq$ $w$.

\section{The spaces $V_{I}(L)$ and $W_{I}(R(k), L)$}

\subsection{The structure of $W_{I}(R(k), L)$}

Theorem 4 ([5]) Let $L \in \mathcal{L}$ and Gosper's equation, corresponding to $L$, have a solution $R(k) \in K(k), \operatorname{den}(R)=g(k)$. Then

$$
W_{I}(R(k), L)=g(k) \cdot V_{I}(\operatorname{pp}(L \circ g(k)))
$$

where the operator $\operatorname{pp}(L \circ g(k))$ is computed by removing from $L \circ g$ the greatest common polynomial factor of its coefficients.

In addition, if $R=\frac{f(k)}{g(k)}, f(k) \perp g(k)$, then the space of the corresponding primitives of the elements of $W_{I}(R(k), L)$ can be described as $f(k) \cdot V_{I}(\operatorname{pp}(L \circ$ $g(k)))$.

We will denote by $\bar{L}$ the operator $\operatorname{pp}(L \circ g(k))$.

Example 6 Consider again the operator $L=k E-(k+1)^{2}$ from Example 1 with $I=\mathbb{Z}$. We have $R=\frac{1}{k}$, and

$L \circ k=k E \circ k-(k+1)^{2} k=k(k+1) E-(k+1)^{2} k=k(k+1)(E-k-1)$, 


$$
\bar{L}=E-(k+1)
$$

The space $W_{I}(R(k), \bar{L})$ is generated by $\bar{t}_{2}$, and, resp., the space $k$. $W_{I}(R(k), \bar{L})$ is generated by $k \bar{t}_{2}$. In accordance with Theorem 4 the space $W_{I}(R(k), L)$ coincides with $k \cdot V_{I}(\bar{L})$.

It is possible to give examples showing that in some cases $\operatorname{dim} W_{I}(R(k), L)>1$.

Example 7 Let $L=2\left(k^{2}-4\right)(k-9) E-(2 k-3)(k-1)(k-8), I=\mathbb{Z}$. Then Gosper's equation, corresponding to $L$, has the rational solution

$$
R(k)=-\frac{2(k-3)(k+1)}{k-9} .
$$

Here $g(k)=k-9$ and $\bar{L}=2\left(k^{2}-4\right) E-(2 k-3)(k-1)$. Any sequence $\bar{t}$ which satisfies the equation $\bar{L} \bar{t}=0$ has $\bar{t}(k)=0$ for $k=2$ or $k \leq-2$. The values of $\bar{t}(1)$ and $\bar{t}(3)$ can be chosen arbitrarily, and all the other values are determined uniquely by the recurrence $2\left(k^{2}-4\right) \bar{t}(k+1)=(2 k-3)(k-1) \bar{t}(k)$. Hence $\operatorname{dim} V_{I}(\bar{L})=2$.

At the same time, $\operatorname{dim} V_{I}(L)=3$. Indeed, if $L t=0$, then $t(-2)=t(2)=$ $t(9)=0$. The value $t(k)=0$ from $k=-2$ propagates to all $k \leq-2$, but on each of the integer intervals $[-1,0,1],[3,4,5,6,7,8]$ and $[10,11, \ldots)$ we can choose one value arbitrarily, and the remaining values on that interval are then determined uniquely. A sequence $t(k) \in V_{I}(L)$ belongs to $W_{I}(R(k), L)$ iff $22 t(10)-13 t(8)=0$. So $\operatorname{dim} W_{I}(R(k), L)=2$.

\subsection{When a rational solution of Gosper's equation is not unique}

We give an example showing that if $L \in \mathcal{L}$ and Gosper's equation, corresponding to $L$, has different solutions $R_{1}(k), R_{2}(k) \in K(k)$, then it is possible that $W_{I}\left(R_{1}(k), L\right) \neq W_{I}\left(R_{2}(k), L\right)$. Moreover, these two spaces can have different dimensions.

Example 8 If $L=k E-(k+1)$, then Gosper's equation, corresponding to $L$, is

$$
-(k+1) R(k+1)+k R(k)=-k,
$$

and its general rational solution is

$$
\frac{k-1}{2}+\frac{c}{k}=\frac{k^{2}-k+2 c}{2 k} .
$$


Consider the solutions

$$
R_{1}(k)=\frac{k-1}{2}\left(g_{1}(k)=1\right), \text { and } R_{2}(k)=\frac{k^{2}-k+2}{2 k}\left(g_{2}(k)=k\right) .
$$

We have $L \circ g_{1}(k)=L$, and $W_{I}\left(R_{1}(k), L\right)=V_{I}(L)$. This space has a basis that consists of two linearly independent sequences:

$$
t_{1}(k)= \begin{cases}k, & \text { if } k \leq 0 \\ 0, & \text { if } k>0\end{cases}
$$

and

$$
t_{2}(k)= \begin{cases}0, & \text { if } k \leq 0 \\ k, & \text { if } k>0\end{cases}
$$

So this space contains, e.g., the sequence $t(k)=|k|$.

We have $L \circ g_{2}(k)=k(k+1)(E-1)$, therefore $W_{I}\left(R_{2}(k), L\right)$ is generated by the sequence $t(k)=k$.

If Gosper's equation, corresponding to $L \in \mathcal{L}$, has non-unique solution in $K(k)$, then the equation $L y=0$ has a non-zero solution in $K(k)$.

\subsection{If Gosper's equation has a rational solution $R(k)$ then $W_{I}(R, L) \neq 0$}

Theorem 5 ([5]) Let $L \in \mathcal{L}$ and let Gosper's equation, corresponding to $L$, have a solution $R(k) \in K(k)$. Then $W_{I}(R(k), L) \neq 0$ (i.e., $\left.\operatorname{dim} W_{I}(R(k), L) \geq 1\right)$.

Example 9 Let $L=(k+2) E-k$. The rational function $\frac{1}{k(k+1)}$ is a solution in $K(k)$ of the equation $L y=0$. Here $R(k)=-k-1$, and $-1 / k$ is a solution of the corresponding telescoping equation:

$$
-\frac{1}{k+1}+\frac{1}{k}=\frac{1}{k(k+1)}
$$

The rational functions

$$
\frac{1}{k(k+1)} \text { and }-\frac{1}{k}
$$

have integer poles. Nevertheless, by Theorem 5 it has to be $W_{I}(R(k), L) \neq 0$ even when $I=\mathbb{Z}$. The space $W_{I}(R(k), L)$ is generated by the sequence

$$
t(k)= \begin{cases}1, & \text { if } k=-1 \\ -1, & \text { if } k=0 \\ 0, & \text { otherwise }\end{cases}
$$


while the primitive of $t(k)$ is

$$
(-k-1) t(k)= \begin{cases}1, & \text { if } k=0 \\ 0, & \text { otherwise }\end{cases}
$$

If $I=\mathbb{Z}_{\geq 1}$, then $W_{I}(R(k), L)$ is generated by the sequence $t^{\prime}(k)=\frac{1}{k(k+1)}$.

By Theorem 3 , if $M$ is the maximal integer singularity of $L, l \geq M$, $I=\mathbb{Z}_{\geq l}$, and Gosper's equation, corresponding to $L$, has a solution $R(k)$ in $K(\bar{k})$, then $V_{I}(L)=W_{I}(R(k), L)$. As a consequence, $\operatorname{dim} V_{I}(L)=$ $\operatorname{dim} W_{I}(R(k), L)=1$.

\section{References}

[1] S. A. Abramov, On the summation of rational function, USSR Comput. Math. Phys. 11 (1971), 324-330. Transl. from Zh. vychisl. mat. mat. fyz. 11 (1971), 1071-1075.

[2] S. A. Abramov, Rational solutions of linear difference and differential equations with polynomial coefficients, USSR Comput. Math. Phys. 29 (1989), 7-12. Transl. from Zh. vychisl. mat. mat. fyz. 29 (1989), 16111620 .

[3] S. A. Abramov, Rational solutions of linear difference and $q$-difference equations with polynomial coefficients, Programming and Comput. Software 21 (1995), 273-278. Transl. from Programmirovanie 21 (1995), $3-11$.

[4] S. A. Abramov and M. Petkovšek, Gosper's Algorithm, Accurate Summation, and the discrete Newton-Leibniz formula, ISSAC'05 (Annual International Symposium on Symbolic and Algebraic Computation). Beijing, China; 24-27 July 2005; Proceedings ISSAC'05, 5-12.

[5] S. A. Abramov, On the summation of $P$-recursive sequences, ISSAC'06 (Annual International Symposium on Symbolic and Algebraic Computation). Genova, Italy; 9-12 July 2006; Proceedings ISSAC'06, 17-22.

[6] R. W. Gosper, Jr., Decision procedure for indefinite hypergeometric summation, Proc. Natl. Acad. Sci. USA 75 (1978), 40-42. 